DOI: $10.2478 / \mathrm{v} 10324-012-0001-8$

Analele Universităţii de Vest, Timişoara

Seria Matematică - Informatică

VERSITA

L, 1, (2012), 03- 25

\title{
On the $\Psi-$ Asymptotic Stability of Nonlinear Lyapunov Matrix Differential Equations
}

Aurel Diamandescu

\begin{abstract}
In this paper are proved (necessary and) sufficient conditions for $\Psi$ - asymptotic stability of the trivial solution of nonlinear Lyapunov matrix differential equations.
\end{abstract}

AMS Subject Classification (2000). 34D20, 34D05.

Keywords. $\Psi-$ asymptotic stability, Lyapunov matrix differential equation.

\section{Introduction}

The Lyapunov matrix differential equations occur in many branches of control theory, such as optional control and stability analysis. Two point boundary value problems for the equations (1) and (3) were studied in many papers ([8] - [12]). Recent works for $\Psi$ - boundedness and $\Psi$ - stability, dichotomy and conditioning for Lyapunov matrix differential equations have been given by M.S.N. Murty, G. Suresh Kumar and A. Diamandescu in [5], [13], [14].

In our paper [6] have been proved (necessary and) sufficient conditions for $\Psi-$ (uniform) stability of the trivial solutions of (nonlinear) Lyapunov matrix differential equations (1), (2) and (3).

The purpose of this paper is to prove (necessary and) sufficient conditions for $\Psi-$ asymptotic stability of trivial solution of the nonlinear Lyapunov matrix differential equation

$$
Z^{\prime}=A(t) Z+Z B(t)+F(t, Z)
$$


and the linear equation

$$
Z^{\prime}=\left[A(t)+A_{1}(t)\right] Z+Z\left[B(t)+B_{2}(t)\right]
$$

which can be seen as a perturbed version of the linear equation

$$
Z^{\prime}=A(t) Z+Z B(t)
$$

We investigate conditions on the fundamental matrices of the equations

$$
\begin{aligned}
& X^{\prime}=A(t) X \\
& Y^{\prime}=Y B(t)
\end{aligned}
$$

and on the functions $A_{1}, B_{1}$ and $F$ under which the trivial solution of the equations (1), (2) and (3) is $\Psi$ - asymptotically stable on $\mathbb{R}_{+}$. Here, $\Psi$ is a matrix function whose introduction permits us obtaining a mixed asymptotic behavior for the components of solutions.

The main tool used in this paper is the technique of Kronecker product of matrices, which has been successfully applied in various fields of matrix theory, group theory and particle physics. See, for example, the above cited papers.

\section{Preliminaries}

In this section we present some basic definitions, notations, hypotheses and results which are useful later on. Let $\mathbb{R}^{n}$ be the Euclidean $n$-dimensional space. For $x=\left(x_{1}, x_{2}, x_{3}, \ldots, x_{n}\right)^{T} \in \mathbb{R}^{n}$, let $\|x\|=\max \left\{\left|x_{1}\right|,\left|x_{2}\right|,\left|x_{3}\right|, \ldots,\left|x_{\mathrm{n}}\right|\right\}$ be the norm of $x\left({ }^{T}\right.$ denotes transpose $)$.

Let $\mathbb{M}_{m \times n}$ be the linear space of all $m \times n$ real valued matrices.

For a matrix $A=\left(a_{i j}\right) \in \mathbb{M}_{n \times n}$, we define the norm $|A|$ by $|A|=\sup _{\|x\| \leq 1}\|A x\|$. It is well-known that $|A|=\max _{1 \leq i \leq n}\left\{\sum_{j=1}^{n}\left|a_{i j}\right|\right\}$.

Definition 1. ([[1]) Let $A=\left(a_{i j}\right) \in \mathbb{M}_{m \times n}$ and $B=\left(b_{i j}\right) \in \mathbb{M}_{p \times q}$. The Kronecker product of $A$ and $B$, written $A \otimes B$, is defined to be the partitioned matrix

$$
A \otimes B=\left(\begin{array}{cccc}
a_{11} B & a_{12} B & \cdots & a_{1 n} B \\
a_{21} B & a_{22} B & \cdots & a_{2 n} B \\
\vdots & \vdots & \vdots & \vdots \\
a_{m 1} B & a_{m 2} B & \cdots & a_{m n} B
\end{array}\right)
$$


Obviously, $A \otimes B \in \mathbb{M}_{m p} \times n q$.

Lemma 1. The Kronecker product has the following properties and rules, provided that the dimension of the matrices are such that the various expressions exist:

1). $A \otimes(B \otimes C)=(A \otimes B) \otimes C$;

2). $(A \otimes B)^{T}=A^{T} \otimes B^{T}$;

3). $(A \otimes B) \cdot(C \otimes D)=(A \cdot C) \otimes(B \cdot D)$;

4). $(A \otimes B)^{-1}=A^{-1} \otimes B^{-1}$;

5). $A \otimes(B+C)=A \otimes B+A \otimes C$;

6). $(A+B) \otimes C=A \otimes C+B \otimes C$;

7). $I_{p} \otimes A=\left(\begin{array}{cccc}A & O & \cdots & O \\ O & A & \cdots & O \\ \vdots & \vdots & \vdots & \vdots \\ O & O & \cdots & A\end{array}\right)$;

8). $(A(t) \otimes B(t))^{\prime}=A^{\prime}(t) \otimes B(t)+A(t) \otimes B^{\prime}(t)$; (here, ' denotes the derivative $\left.\frac{d}{d t}\right)$.

Proof. See in [1]].

Definition 2. The application Vec $: \mathbb{M}_{m \times n} \longrightarrow \mathbb{R}^{m n}$, defined by

$$
\operatorname{Vec}(A)=\left(a_{11}, a_{21}, \cdots, a_{m 1}, a_{12}, a_{22}, \cdots, a_{m 2}, \cdots, a_{1 n}, a_{2 n}, \cdots, a_{m n}\right)^{T},
$$

where $A=\left(a_{i j}\right) \in \mathbb{M}_{m \times n}$, is called the vectorization operator.

Lemma 2. ([6]) The vectorization operator Vec $: \mathbb{M}_{n \times n} \longrightarrow \mathbb{R}^{n^{2}}$, is a linear and one-to-one operator. In addition, Vec and $\mathrm{Vec}^{-1}$ are continuous operators.

Lemma 3. A function $F: \mathbb{R}_{+} \longrightarrow \mathbb{M}_{n \times n}$ is a continuous (differentiable) matrix function on $\mathbb{R}_{+}$if and only if the function $f=\operatorname{Vec}(F): \mathbb{R}_{+} \longrightarrow \mathbb{R}^{n^{2}}$ is a continuous (differentiable) vector function on $\mathbb{R}_{+}$.

Proof. It is a simple exercise.

We recall that the vectorization operator $\mathcal{V e c}$ has the following properties as concerns the calculations (see in [13]):

Lemma 4. If $A, B, M \in \mathbb{M}_{n \times n}$, then

1). $\operatorname{Vec}(A M B)=\left(B^{T} \otimes A\right) \cdot \operatorname{Vec}(M)$;

2). $\operatorname{Vec}(M B)=\left(B^{T} \otimes I_{n}\right) \cdot \operatorname{Vec}(M)$;

3). $\operatorname{Vec}(A M)=\left(I_{n} \otimes A\right) \cdot \operatorname{Vec}(M)$;

4). $\operatorname{Vec}(A M)=\left(M^{T} \otimes A\right) \cdot \operatorname{Vec}\left(I_{n}\right)$. 
Proof. It is a simple exercise.

In the system (1) we assume that $A$ and $B$ are continuous $n \times n$ matrices on $\mathbb{R}_{+}=$ $[0, \infty)$ and $F: \mathbb{R}_{+} \times \mathbb{M}_{n \times n} \longrightarrow \mathbb{M}_{n \times n}$ is a continuous $n \times n$ matrix function such that $F\left(t, O_{n}\right)=O_{n}$ (null matrix of order $n \times n$ ).

By a solution of the equation (1) we mean a continuous differentiable $n \times n$ matrix function satisfying the equation (1) for all $t \geq 0$.

Let $\Psi_{\mathrm{i}}: \mathbb{R}_{+} \longrightarrow(0, \infty), i=1,2, \ldots, n$, be continuous functions and

$$
\Psi=\operatorname{diag}\left[\Psi_{1}, \Psi_{2}, \cdots \Psi_{n}\right]
$$

Definition 3. ([4]) The trivial solution of the vector differential equation $\quad x^{\prime}=$ $f(t, x)$ (where $x \in \mathbb{R}^{n}$ and $f$ is a continuous $n$ vector function) is said to be $\Psi-$ stable on $\mathbb{R}_{+}$iffor every $\varepsilon>0$ and every $t_{0} \in \mathbb{R}_{+}$, there exists $\delta=\delta\left(\varepsilon, t_{0}\right)>0$ such that any solution $x(t)$ of the equation which satisfies the inequality $\left\|\Psi\left(t_{0}\right) x\left(t_{0}\right)\right\|<$ $\delta$, exists and satisfies the inequality $\|\Psi(t) x(t)\|<\varepsilon$ for all $t \geq t_{0}$.

The trivial solution of the vector differential equation $x^{\prime}=f(t, x)$ is said to be $\Psi$ uniformly stable on $\mathbb{R}_{+}$if it is $\Psi$ - stable on $\mathbb{R}_{+}$and the above $\delta$ is independent of $t_{0}$.

Definition 4. ([4]) The trivial solution of the vector differential equation $x^{\prime}=$ $f(t, x)$ is said to be $\Psi$ - asymptotically stable on $\mathbb{R}_{+}$if it is $\Psi$ - stable on $\mathbb{R}_{+}$and in addition, for every $t_{0} \in \mathbb{R}_{+}$, there exists $\delta_{0}=\delta_{0}\left(t_{0}\right)>0$ such that any solution $x(t)$ of the equation which satisfies the inequality $\left\|\Psi\left(t_{0}\right) x\left(t_{0}\right)\right\|<\delta_{0}$, satisfies the condition $\lim _{t \rightarrow \infty} \Psi(t) x(t)=0$.

Extend these definitions for a matrix differential equation $X^{\prime}=F(t, X)$, where $X \in \mathbb{M}_{n \times n}$ and $F$ is a continuous $n \times n$ matrix function.

Definition 5. The trivial solution of the matrix differential equation $X^{\prime}=F(t, X)$ is said to be $\Psi$ - stable on $\mathbb{R}_{+}$iffor every $\varepsilon>0$ and every $t_{0} \in \mathbb{R}_{+}$, there exists $\delta=\delta\left(\varepsilon, t_{0}\right)>0$ such that any solution $X(t)$ of the equation which satisfies the inequality $\left|\Psi\left(t_{0}\right) X\left(t_{0}\right)\right|<\delta$, exists and satisfies the inequality $|\Psi(t) X(t)|<\varepsilon$ for all $t \geq t_{0}$.

The trivial solution of the matrix differential equation $X^{\prime}=F(t, X)$ is said to be $\Psi$ - uniformly stable on $\mathbb{R}_{+}$if it is $\Psi$ - stable on $\mathbb{R}_{+}$and the above $\delta$ is independent of $t_{0}$.

Definition 6. The trivial solution of the matrix differential equation $X^{\prime}=F(t, X)$ is said to be $\Psi$ - asymptotically stable on $\mathbb{R}_{+}$if it is $\Psi$ - stable on $\mathbb{R}_{+}$and in addition, for every $t_{0} \in \mathbb{R}_{+}$, there exists $\delta_{0}=\delta_{0}\left(t_{0}\right)>0$ such that any solution 
$X(t)$ of the equation which satisfies the inequality $\left|\Psi\left(t_{0}\right) X\left(t_{0}\right)\right|<\delta_{0}$, satisfies the condition $\lim _{t \rightarrow \infty} \Psi(t) X(t)=O_{n}$.

The following lemmas play a vital role in the proofs of main results.

Lemma 5. ([6]) The matrix function $Z(t)$ is a solution of (1) on the interval $J \subset \mathbb{R}_{+}$if and only if the vector valued function $z(t)=$ Vec $Z(t)$ is a solution of the differential system

$$
z^{\prime}=\left(I_{n} \otimes A(t)+B^{T}(t) \otimes I_{n}\right) z+f(t, z)
$$

where $f(t, z)=\operatorname{Vec} F(t, Z)$, on the same interval $J$.

Definition 7. The above system (6) is called "corresponding Kronecker product system associated with (1)”.

Lemma 6. ([[]]) For every matrix function $M: \mathbb{R}_{+} \longrightarrow \mathbb{M}_{n \times n}$, we have

$$
\frac{1}{n}|\Psi(t) M(t)| \leq\left\|\left(I_{n} \otimes \Psi(t)\right) \operatorname{Vec} M(t)\right\|_{\mathbb{R}^{n^{2}}} \leq|\Psi(t) M(t)|, t \geq 0 .
$$

Lemma 7. The trivial solution of the equation (1) is $\Psi$-asymptotically stable on $\mathbb{R}_{+}$if and only if the trivial solution of the corresponding Kronecker product system associated with (1) - i.e. the system (6) - is $I_{n} \otimes \Psi$ - asymptotically stable on $\mathbb{R}_{+}$.

Remark. The Lemma 7, [6], is a similar result for $\Psi-$ (uniform) stability of the trivial solution of the equation (1).

Proof of Lemma 7. First, suppose that the trivial solution of the equation (1) is $\Psi$ - asymptotically stable on $\mathbb{R}_{+}$. From the above Definition 6 and Lemma 7 , [6], it follows that the trivial solution of the system (6) (i.e. the corresponding Kronecker product system associated with (1)) is $I_{n} \otimes \Psi$ - stable on $\mathbb{R}_{+}$.

According to Definition 6 again, for a given $t_{0} \in \mathbb{R}_{+}$, we choose $\delta_{0}^{\prime}\left(t_{0}\right)=\frac{1}{n} \delta_{0}\left(t_{0}\right)$. Let $z(t)$ be a solution of (6) such that $\left\|\left(I_{n} \otimes \Psi\left(t_{0}\right)\right) z\left(t_{0}\right)\right\|_{\mathbb{R}^{n^{2}}}<\delta_{0}^{\prime}\left(t_{0}\right)$. From Lemma 5 and Lemma 6 , the matrix function $Z(t)=\mathcal{V e c}{ }^{-1} z(t)$ is a solution on $\mathbb{R}_{+}$ of the equation (1) such that $\left|\Psi\left(t_{0}\right) Z\left(t_{0}\right)\right|<\delta_{0}\left(t_{0}\right)$. It follows that $\lim _{t \rightarrow \infty} \Psi(t) X(t)=$ $O_{n}$.

From Lemma 6 again, we have that $\lim _{t \rightarrow \infty}\left(I_{n} \otimes \Psi(t)\right) z(t)=0$. Thus, the trivial solution of the system (6) is $I_{n} \otimes \Psi$ - asymptotically stable on $\mathbb{R}_{+}$. 
Suppose, conversely, that the trivial solution of the system (6) is $I_{n} \otimes \Psi$ - asymptotically stable on $\mathbb{R}_{+}$. From Definition 4 and Lemma 7, [6], it follows that the trivial solution of the equation (1) is $\Psi-$ stable on $\mathbb{R}_{+}$.

According to Definition 4 again, for a given $t_{0} \in \mathbb{R}_{+}$, we choose $\delta_{0}^{\prime}\left(t_{0}\right)=\delta_{0}\left(t_{0}\right)$. Let $Z(t)$ a solution of (1) such that $\left|\Psi\left(t_{0}\right) Z\left(t_{0}\right)\right|<\delta_{0}^{\prime}$. From Lemma 5 and Lemma 6, the function $z(t)=\operatorname{Vec} Z(t)$ is a solution on $\mathbb{R}_{+}$of the system (6) such that $\left\|\left(I_{n} \otimes \Psi\left(t_{0}\right)\right) z\left(t_{0}\right)\right\|_{\mathbb{R}^{n^{2}}}<\delta_{0}\left(t_{0}\right)$. It follows that $\lim _{t \rightarrow \infty}\left(I_{n} \otimes \Psi(t)\right) z(t)=0$. From Lemma 6 again, we have that $\lim _{t \rightarrow \infty} \Psi(t) Z(t)=O_{n}$. Thus, the trivial solution of the equation (1) is $\Psi$ - asymptotically stable on $\mathbb{R}_{+}$.

The proof is now complete.

Lemma 8. ([6] Let $X(t)$ and $Y(t)$ be fundamental matrices for the equations (4) and (5) respectively. Then, the matrix $Z(t)=Y^{T}(t) \otimes X(t)$ is a fundamental matrix for the system

$$
z^{\prime}=\left(I_{n} \otimes A(t)+B^{T}(t) \otimes I_{n}\right) z
$$

Remark. The above result is Lemma 1 of [13]. Because the proof is incomplete, we presented it with a complete proof.

\section{$3 \Psi-$ asymptotic stability of the linear Lyapunov matrix dif- ferential equations}

The purpose of this section is to give conditions for $\Psi$ - asymptotic stability of trivial solutions of linear Lyapunov matrix differential equations (2) and (3). These conditions can be expressed in terms of a fundamental matrices for the equations (4) and (5).

Theorem 1. Let $X(t)$ and $Y(t)$ be a fundamental matrices for the equations (4) and (5) respectively. Then, the trivial solution of (3) is $\Psi$-asymptotically stable on $\mathbb{R}_{+}$if and only if

$$
\lim _{t \rightarrow \infty} Y^{T}(t) \otimes \Psi(t) X(t)=O_{n^{2}} .
$$

Proof. First, suppose that the trivial solution of the equation (3) is $\Psi$ - asymptotically stable on $\mathbb{R}_{+}$. From Lemma 7 , it follows that the trivial solution of the corresponding Kronecker product system

$$
z^{\prime}=\left(I_{n} \otimes A(t)+B^{T}(t) \otimes I_{n}\right) z
$$


is $I_{n} \otimes \Psi$ - asymptotically stable on $\mathbb{R}_{+}$. From Theorem 1 , [4], it follows that a fundamental matrix $Z(t)$ of (9) satisfies the condition

$$
\lim _{t \rightarrow \infty}\left(I_{n} \otimes \Psi(t)\right) Z(t)=O_{n^{2}}
$$

From Lemma 8, we replace $Z(t)=Y^{T}(t) \otimes X(t)$ as a fundamental matrix on $\mathbb{R}_{+}$ for the system (9). After computation, it follows that (8) holds.

Now, suppose that (8) holds. This shows that the fundamental matrix $Z(t)=$ $Y^{T}(t) \otimes X(t)$ of the system (9) satisfies the condition (10). From Theorem 1, [4], it follows that the trivial solution of the system (9) is $I_{n} \otimes \Psi$ - asymptotically stable on $\mathbb{R}_{+}$. From Lemma 7, it follows that the trivial solution of the equation (3) is $\Psi$ - asymptotically stable on $\mathbb{R}_{+}$.

The proof is now complete.

Remark. 1 . In the same manner as in classical stability, we can speak about $\Psi-$ asymptotic stability of the linear differential equation (3).

2. It is easy to see that if $\Psi$ and $\Psi^{-1}$ are bounded on $\mathbb{R}_{+}$, then the $\Psi$ - asymptotic stability of the linear equation (3) is equivalent with the classical asymptotic stability of the linear equation (3).

Remark. The Theorem generalizes Theorem 1, [4].

Indeed, in the particular case $B(t)=O_{n}$, we have $Y=I_{n}$ and then $Z(t)=I_{n} \otimes X(t)$. On the other hand, it is easy to see that the solutions of (3) are $Z(t)=X(t) C, C$ being a $n \times n$ constant matrix. In this case, the condition (8) becomes the condition from Theorem 1, [4], concerning $\Psi$ - asymptotic stability of the linear system $x^{\prime}=A(t) x$, because $\left|I_{n} \otimes M\right|=|M|$

Corollary. Suppose that it is satisfied either one or the other of conditions

a). the equation (4) is $\Psi$-asymptotically stable on $\mathbb{R}_{+}$and the equation (5) is stable on $\mathbb{R}_{+}$;

b). the equation (4) is $\Psi$ - stable on $\mathbb{R}_{+}$and the equation (5) is asymptotically stable on $\mathbb{R}_{+}$.

Then, the equation (3) is $\Psi$ - asymptotically stable on $\mathbb{R}_{+}$.

Proof. It results from the inequality $|A \otimes B| \leq|A||B|$, for all $A, B \in \mathbb{M}_{n \times n}$ and from Theorem 1, [6], Theorem 1, [4] and the above Theorem.

Sufficient conditions for the $\Psi$ - asymptotic stability of a linear Lyapunov matrix differential equation (3) are given by the next theorems.

Theorem 2. Let $X(t)$ and $Y(t)$ be fundamental matrices for the equations (4) and (5) respectively. Suppose that there exist the constants $p \geq 1$ and $M>0$ and $a$ 
continuous function $\varphi: \mathbb{R}_{+} \longrightarrow(0, \infty)$ with $\int_{0}^{\infty} \varphi(s) d s=+\infty$ which fulfill one of the following conditions:

$$
\text { (i). } \int_{0}^{t} \varphi(s)\left|\left(Y^{T}(t)\left(Y^{T}(s)\right)^{-1}\right) \otimes\left(\Psi(t) X(t) X^{-1}(s) \Psi^{-1}(s)\right)\right|^{p} d s \leq M,
$$

for all $t \geq 0$,

$$
\text { (ii). } \int_{0}^{t} \varphi(s)\left|\left(\left(Y^{T}(s)\right)^{-1} Y^{T}(t)\right) \otimes\left(X^{-1}(s) \Psi^{-1}(s) \Psi(t) X(t)\right)\right|^{p} d s \leq M,
$$

for all $t \geq 0$.

Then, the equation (3) is $\Psi$-asymptotically stable on $\mathbb{R}_{+}$.

Proof. In the same manner as in the proof of Theorem 2, [4], it follows that there exists a positive constant $K$ such that

$$
\mid\left(Y^{T}(t) \otimes \Psi(t) X(t) \mid \leq K e^{-(p M)^{-1} \int_{1}^{t} \varphi(s) d s}, t \geq 1 .\right.
$$

From Theorem 1, it follows that the system (3) is $\Psi$ - asymptotically stable on $\mathbb{R}_{+}$.

Remark. 1. The Theorem generalizes Theorem 2, [4].

2. The function $\varphi$ can serve to weaken the required hypotheses on the fundamental matrices $X$ and $Y$.

In the conditions of the Theorem, the equation (3) can not be $\Psi$ - uniformly stable on $\mathbb{R}_{+}$. This is shown in the next example, adapted from J. L. Massera and J. J. Schäffer, [7].

Example 1. Let a(t) be a real, continuously differentiable function, equal to 1 except in the intervals $J_{n}=\left[n-2^{-4 n}, n+2^{-4 n}\right], n=1,2, \ldots ; i n J_{n}, a(t)$ lies between 1 and $4^{n}$ and $a(n)=4^{n}$.

Consider the equation (3) with

$$
A(t)=\left(\begin{array}{cc}
-4-\frac{a^{\prime}(t)}{a(t)} & 0 \\
0 & -1
\end{array}\right), B(t)=\left(\begin{array}{cc}
5 & 10 \\
-3 & -6
\end{array}\right) .
$$

The matrix $B$ has the eigenvalues $\lambda_{1}=0, \lambda_{2}=-1$ and the Jordan canonical form $L=\operatorname{diag}[0,-1]$. We have $B^{T}=U L U^{-1}$, where $U=\left(\begin{array}{ll}-3 & 1 \\ -5 & 2\end{array}\right)$.

The fundamental matrices for the equations (4) and (5) are

$$
X(t)=\left(\begin{array}{cc}
\frac{e^{-4 t}}{a(t)} & 0 \\
0 & e^{-t}
\end{array}\right)
$$


Vol. L (2012) On the $\Psi$ - Asymptotic Stability of Nonlinear Lyapunov...

and

$$
Y^{T}(t)=U e^{L t} U^{-1}=\left(\begin{array}{cc}
6-5 e^{-t} & 3 e^{-t}-3 \\
10-10 e^{-t} & 6 e^{-t}-5
\end{array}\right)
$$

respectively.

Consider

$$
\Psi(t)=\left(\begin{array}{cc}
e^{2 t} & 0 \\
0 & e^{-t}
\end{array}\right)
$$

For $\mathrm{t} \geq \mathrm{s} \geq 0$, we have

$$
\Psi(t) X(t) X^{-1}(s) \Psi^{-1}(s)=\left(\begin{array}{cc}
\frac{a(s)}{a(t)} e^{-2(t-s)} & 0 \\
0 & e^{-2(t-s)}
\end{array}\right)
$$

and

$$
Y^{T}(t)\left(Y^{T}(s)\right)^{-1}=\left(\begin{array}{cc}
6-5 e^{-(t-s)} & 3 e^{-(t-s)}-3 \\
10-10 e^{-(t-s)} & 6 e^{-(t-s)}-5
\end{array}\right) .
$$

It follows that

$$
\begin{gathered}
\left|\left(Y^{T}(t)\left(Y^{T}(s)\right)^{-1}\right) \otimes\left(\Psi(t) X(t) X^{-1}(s) \Psi^{-1}(s)\right)\right| \leq \\
\quad \leq 21 \max \left\{\frac{a(s)}{a(t)} e^{-2(t-s)}, e^{-2(t-s)}\right\}, t \geq s \geq 0,
\end{gathered}
$$

and then

$$
\int_{0}^{t}\left|\left(Y^{T}(t)\left(Y^{T}(s)\right)^{-1}\right) \otimes\left(\Psi(t) X(t) X^{-1}(s) \Psi^{-1}(s)\right)\right| d s \leq 25, \text { for all } t \geq 0 .
$$

Thus, the condition (i) of the Theorem is satisfied with $\varphi=1, p=1, M=25$.

It follows that the equation (3) is $\Psi$ - asymptotically stable on $\mathbb{R}_{+}$.

On the other hand, for $s=n, t=n+2^{-4 n}$ we have

$$
\begin{gathered}
\left|\left(Y^{T}(t)\left(Y^{T}(s)\right)^{-1}\right) \otimes\left(\Psi(t) X(t) X^{-1}(s) \Psi^{-1}(s)\right)\right| \geq \\
\geq\left|\left(6-5 e^{-2^{-4 n}}\right) 4^{n} e^{-2^{-4 n+1}}\right| \longrightarrow \infty .
\end{gathered}
$$

From Theorem 1 [6], it follows that the equation (3) is not $\Psi$ - uniformly stable on $\mathbb{R}_{+}$.

Theorem 3. Suppose that $A_{1}, B_{1}$ are continuous $n \times n$ matrices on $\mathbb{R}_{+}$. If the linear Lyapunov matrix differential equation (3) is $\Psi$ - uniformly and asymptotically stable on $\mathbb{R}_{+}$and if

$$
\int_{0}^{\infty}\left|I_{n} \otimes\left(\Psi(t) A_{1}(t) \Psi^{-1}(t)\right)+B_{1}^{T}(t) \otimes I_{n}\right| d t<+\infty
$$


then, the linear Lyapunov matrix differential equation (2) is also $\Psi$ - uniformly and asymptotically stable on $\mathbb{R}_{+}$.

Proof. The corresponding Kronecker product system associated with (2) can be written in the form

$$
z^{\prime}=\left(I_{n} \otimes A(t)+B^{T}(t) \otimes I_{n}\right) z+\left(I_{n} \otimes A_{1}(t)+B_{1}^{T}(t) \otimes I_{n}\right) z,
$$

i.e. as a perturbed system of the corresponding Kronecker product system associated with (3),

$$
z^{\prime}=\left(I_{n} \otimes A(t)+B^{T}(t) \otimes I_{n}\right) z .
$$

From the hypotheses, Lemma 7, [6], and Lemma 7, the system (13) is $\mathrm{I}_{n} \otimes \Psi-$ uniformly and asymptotically stable on $\mathbb{R}_{+}$.

On the other hand, from hypotheses, we have that the matrix $I_{n} \otimes A_{1}(t)+B_{1}^{T}(t)$ $\otimes I_{n}$ satisfies the conditions of Theorem 3, [6] and Theorem 3, [4].

Now, from these Theorems, it follows that the system (12) is $I_{n} \otimes \Psi-$ uniformly and asymptotically stable on $\mathbb{R}_{+}$.

From Lemma 7, [6] and Lemma 7 again, it follows that the equation (2) is $\Psi-$ uniformly and asymptotically stable on $\mathbb{R}_{+}$.

The proof is now complete.

Remark. The Theorem generalizes Theorem 3, [4].

If we require that the linear equation (3) is only $\Psi$ - asymptotically stable on $\mathbb{R}_{+}$, then the above Theorem is no longer true. This is shown by the next Example, transformed after an example due to O. Perron [15].

Example 2. Let $a \in \mathbb{R}$ such that $1<2 a<1+e^{-\pi}$ and the equation (3) with

$$
\mathrm{A}(\mathrm{t})=\left(\begin{array}{lc}
\mathrm{a} & 0 \\
0 & \sin \ln (t+1)+\cos \ln (t+1)
\end{array}\right), B(t)=\left(\begin{array}{cc}
-2 a & 0 \\
0 & -2 a
\end{array}\right) .
$$

Then, a fundamental matrix for the equation (3) is

$$
Z(t)=\left(\begin{array}{cc}
e^{-a(t+1)} & 0 \\
0 & e^{(t+1)(\sin \ln (t+1)-2 a)}
\end{array}\right)
$$

Consider $\Psi(\mathrm{t})=\left(\begin{array}{cc}e^{-\frac{a}{2}(t+1)} & 0 \\ 0 & e^{\frac{2 a-1}{2}(t+1)}\end{array}\right)$.

From $\lim _{t \rightarrow \infty} \Psi(t) Z(t)=0$, it follows that the equation (3) is $\Psi$ - asymptotically stable on $\mathbb{R}_{+}$. 
As in Example 3, [4], we can show that $\Psi(t) Z(t) Z^{-1}(s) \Psi^{-1}(s)$ is not bounded for $0 \leq s \leq t<+\infty$. From Theorem 1, [6], it follows that the equation (3) is not $\Psi-$ uniformly stable on $\mathbb{R}_{+}$.

Now, we take

$$
A_{1}(t)=\left(\begin{array}{cc}
0 & 0 \\
e^{-a(t+1)} & 0
\end{array}\right) \text { and } B_{1}(t)=\left(\begin{array}{ll}
0 & 0 \\
0 & 0
\end{array}\right)
$$

Then, a fundamental matrix for the perturbed equation (2) is

$$
\widetilde{Z}(t)=\left(\begin{array}{cc}
e^{-a(t+1)} & 0 \\
e^{(t+1)(\sin \ln (t+1)-2 a)} \int_{1}^{t+1} e^{-s \sin \ln s} d s & e^{(t+1)(\sin \ln (t+1)-2 a)}
\end{array}\right) .
$$

As in Example 3, [4], we can show that $\lim _{t \rightarrow \infty} \Psi(t) \widetilde{Z}(t) \neq 0$. It follows that the equation (2) is not $\Psi$ - asymptotically stable on $\mathbb{R}_{+}$.

Finally, it has been ascertained that the condition (11) is satisfied.

Also, Theorem 3 is no longer true if we require that

$$
\lim _{t \longrightarrow \infty}\left|I_{n} \otimes\left(\Psi(t) A_{1}(t) \Psi^{-1}(t)\right)+B_{1}^{T}(t) \otimes I_{n}\right|=0,
$$

instead of the condition (11).

This is shown by the next Example, transformed after an example due to L. Cesari [2].

Example 3. Consider the equation (3) with

$$
A(t)=\left(\begin{array}{cc}
\frac{2}{t+1} & 1 \\
-1 & 0
\end{array}\right), B(t)=\left(\begin{array}{cc}
-\frac{2}{t+1} & 0 \\
0 & -\frac{2}{t+1}
\end{array}\right) .
$$

Then, a fundamental matrix for this equation is

$$
Z(t)=\left(\begin{array}{cc}
\frac{\sin (t+1)}{t+1} & \frac{\cos (t+1)}{t+1} \\
\frac{(t+1) \cos (t+1)-\sin (t+1)}{(t+1)^{2}} & -\frac{(t+1) \sin (t+1)+\cos (t+1)}{(t+1)^{2}}
\end{array}\right) .
$$

Consider $\Psi(\mathrm{t})=\left(\begin{array}{cc}\sqrt[3]{t+1} & 0 \\ 0 & \sqrt[3]{t+1}\end{array}\right)$

From

$$
\Psi(t) Z(t)=\left(\begin{array}{cc}
\frac{\sin (t+1)}{\sqrt[3]{(t+1)^{2}}} & \frac{\cos (t+1)}{\sqrt[3]{(t+1)^{2}}} \\
\frac{(t+1) \cos (t+1)-\sin (t+1)}{\sqrt[3]{(t+1)^{5}}} & -\frac{(t+1) \sin (t+1)+\cos (t+1)}{\sqrt[3]{(t+1)^{5}}}
\end{array}\right)
$$


for all $\mathrm{t} \geq 0$ and

$$
\begin{gathered}
\Psi(t) Z(t) Z^{-1}(s) \Psi^{-1}(s)=\frac{\sqrt[3]{t+1}}{\sqrt[3]{s+1}} \cdot \\
\cdot\left(\begin{array}{cc}
\frac{(s+1) \cos (t-s)+\sin (t-s)}{t+1} & \frac{(s+1) \sin (t-s)}{t+1} \\
\frac{(t-s) \cos (t-s)-(t s+t+s+2) \sin (t-s)}{(t+1)^{2}} & \frac{(s+1)(t+1) \cos (t-s)-(s+1) \sin (t-s)}{(t+1)^{2}}
\end{array}\right)
\end{gathered}
$$

for all $0 \leq s \leq t<+\infty$, it follows that the equation (3) is $\Psi-$ uniformly and asymptotically stable on $\mathbb{R}_{+}$.

Now, if we take

$$
A_{1}(t)=\left(\begin{array}{cc}
0 & 0 \\
0 & \frac{2}{t+1}
\end{array}\right) \text { and } B_{1}(t)=\left(\begin{array}{cc}
0 & 0 \\
0 & 0
\end{array}\right)
$$

then, a fundamental matrix for the perturbed equation (2) is

$$
\widetilde{Z}(t)=\left(\begin{array}{cc}
\sin t & \cos t \\
\cos t & -\sin t
\end{array}\right)
$$

From

$$
\Psi(t) \widetilde{Z}(t)=\sqrt[3]{t+1}\left(\begin{array}{cc}
\sin t & \cos t \\
\cos t & -\sin t
\end{array}\right)
$$

for $\mathrm{t} \geq 0$, it follows that the perturbed equation (2) is not $\Psi$ - asymptotically stable on $\mathbb{R}_{+}$.

Finally, we have that the condition (11) is not satisfied, while

$$
\lim _{t \longrightarrow \infty}\left|I_{2} \otimes\left(\Psi(t) A_{1}(t) \Psi^{-1}(t)\right)+B_{1}^{T}(t) \otimes I_{2}\right|=0
$$

is true.

Theorem 4. Suppose that:

1). There exist a continuous function $\varphi: \mathbb{R}_{+} \longrightarrow(0, \infty)$ with $\int_{0}^{\infty} \varphi(s) d s=+\infty$ and a positive constant $M$ such that the fundamental matrices $X$ and $Y$ for the equations (4) and (5) respectively satisfy the condition

$$
\int_{0}^{t} \varphi(s)\left|\left(Y^{T}(t)\left(Y^{T}(s)\right)^{-1}\right) \otimes\left(\Psi(t) X(t) X^{-1}(s) \Psi^{-1}(s)\right)\right| d s \leq M, \forall t \geq 0
$$

2). $A_{1}(t)$ and $B_{1}(t)$ are continuous $n \times n$ matrices on $\mathbb{R}_{+}$such that

$$
\sup _{t \geq 0} \frac{1}{\varphi(t)}\left|I_{n} \otimes\left(\Psi(t) A_{1}(t) \Psi^{-1}(t)\right)+B_{1}^{T}(t) \otimes I_{n}\right|<\frac{1}{M} .
$$

Then, the linear Lyapunov matrix differential equation (2) is $\Psi$-asymptotically stable on $\mathbb{R}_{+}$. 
Proof. The corresponding Kronecker product system associated with (2) can be seen in the form (12), i.e. as a perturbed system of the corresponding Kronecker product system associated with (3), namely the system (13).

From the hypotheses and Theorem 2, it follows that the equation (3) is $\Psi$ - asymptotically stable on $\mathbb{R}_{+}$. Thereafter, from Lemma 7 , it follows that the system (13) is $I_{n} \otimes \Psi$ - asymptotically stable on $\mathbb{R}_{+}$. From Theorem 4, [4], it follows that the system (12) is $I_{n} \otimes \Psi$ - asymptotically stable on $\mathbb{R}_{+}$. From Lemma 7 again, the equation (2) is $\Psi$ - asymptotically stable on $\mathbb{R}_{+}$.

The proof is now complete.

Remark. The Theorem generalizes Theorem 4, [4].

Remark. The condition from the hypothesis 2) can be replaced with the condition

$$
\lim _{t \rightarrow \infty} \frac{1}{\varphi(t)}\left|I_{n} \otimes\left(\Psi(t) A_{1}(t) \Psi^{-1}(t)\right)+B_{1}^{T}(t) \otimes I_{n}\right|=0 .
$$

The Theorem 4 is no longer true if we require that the equation (3) is only $\Psi_{-}$ asymptotically stable on $\mathbb{R}_{+}$, instead of the condition 1 ) of Theorem. This is shown by the next simple example.

Example 4. Consider the equation (3) with $A(t)=B(t)=O_{2}$. Then, fundamental matrices for the equations (5) and (6) are $X(t)=I_{2}, Y(t)=I_{2}$ respectively.

Consider $\Psi(\mathrm{t})=\left(\begin{array}{cc}\frac{1}{t^{2}+1} & 0 \\ 0 & \frac{1}{t+1}\end{array}\right)$.

Therefore, for a fundamental matrix $\mathrm{Z}$ for the equation (3) we have $\lim _{t \rightarrow \infty} \Psi(t) Z(t)=$ $\mathrm{O}_{2}$.

It follows that the equation (3) is $\Psi$ - asymptotically stable on $\mathbb{R}_{+}$.

Now, we consider the equation (2) with

$$
\mathrm{A}_{1}(\mathrm{t})=\left(\begin{array}{cc}
0 & 0 \\
0 & \frac{a}{\sqrt{t+1}}
\end{array}\right) \text { and } \mathrm{B}_{1}(\mathrm{t})=\mathrm{O}_{2},
$$

a being a positive constant.

It is easy to see (by reduction to absurdity) that there is not a function $\varphi$ that to satisfy the conditions of Theorem 4.

The solutions of the equation (2) are

$$
Z(t)=\left(\begin{array}{cc}
1 & 0 \\
0 & e^{2 a \sqrt{t+1}}
\end{array}\right) \cdot C,
$$

where $C$ is a $2 \times 2$ constant matrix. 
We have

$$
\Psi(t) Z(t)=\left(\begin{array}{cc}
\frac{1}{t^{2}+1} & 0 \\
0 & \frac{e^{2 a \sqrt{t+1}}}{t+1}
\end{array}\right) \cdot C
$$

and then, the perturbed equation (2) is not $\Psi$ - asymptotically stable on $\mathbb{R}_{+}$.

Finally, we have

$$
\sup _{t \geq 0}\left|I_{2} \otimes\left(\Psi(t) A_{1}(t) \Psi^{-1}(t)\right)+B_{1}^{T}(t) \otimes I_{2}\right|=a
$$

and

$$
\lim _{t \longrightarrow \infty}\left|I_{2} \otimes\left(\Psi(t) A_{1}(t) \Psi^{-1}(t)\right)+B_{1}^{T}(t) \otimes I_{2}\right|=0
$$

\section{$4 \Psi$-asymptotic stability of the nonlinear Lyapunov matrix differential equations}

The purpose of this section is to study the $\Psi$ - asymptotic stability of trivial solution of the equation (1), where $A, B$ and $F$ are matrix functions. It will be assumed that $A$ and $B$ are continuous for $t \in \mathbb{R}_{+}$and that $F$ is continuous for $t \in \mathbb{R}_{+}$and $Z \in \mathbb{M}_{n \times n}$. This will ensure the local existence of a solution passing through any given point $\left(t_{0}, Z_{0}\right)$ of the domain of definition of $F$, but it does not guarantee that the solution is unique or that it can be continued for all large values of $t$.

Thus, we state the following hypothesis:

(H): For all $t_{0} \in \mathbb{R}_{+}$and $Z_{0} \in \mathbb{M}_{n \times n}$, there exists a unique solution $Z(t)$ of the equation (1) such that $Z\left(t_{0}\right)=Z_{0}$.

Theorem 5. Suppose that:

1). the hypothesis $(H)$ is satisfied;

2 ). the trivial solution of (4) is $\Psi$ - uniformly and asymptotically stable on $\mathbb{R}_{+}$;

$3)$. the matrix function $F$ satisfies the inequality

$$
|\Psi(t) F(t, Z)| \leq \gamma(t)|\Psi(t) Z|
$$

for all $t \in \mathbb{R}_{+}$and for all $Z \in \mathbb{M}_{n \times n}$, where $\gamma: \mathbb{R}_{+} \longrightarrow \mathbb{R}_{+}$is a continuous function on $\mathbb{R}_{+}$such that $L=\int_{0}^{\infty} \gamma(t) d t<+\infty$.

4). the matrix function $B$ satisfies the condition $L^{\prime}=\int_{0}^{\infty}|B(t)| d t<+\infty$.

Then, the trivial solution of the equation (1) is $\Psi$ - uniformly and asymptotically stable on $\mathbb{R}_{+}$. 
Proof. For all $t_{0} \in \mathbb{R}_{+}$and $Z_{0} \in \mathbb{M}_{n \times n}$, let $Z(t)$ be the unique solution of (1) with $Z\left(t_{0}\right)=Z_{0}$. From Lemma 5, it follows that $z(t)=\mathcal{V} e c Z(t)$ is the unique solution of the system

$$
z^{\prime}=\left(I_{n} \otimes A(t)+B^{T}(t) \otimes I_{n}\right) z+f(t, z)
$$

with $z\left(t_{0}\right)=z_{0}=\operatorname{Vec} Z_{0}$, where $f(t, z)=\operatorname{Vec} F(t, Z)$.

Therefore, $z(t)$ is also a solution of the inhomogeneous linear system

$$
z^{\prime}=I_{n} \otimes A(t) z+\left(\left(B^{T}(t) \otimes I_{n}\right) z(t)+f(t, z(t))\right), t \in\left[t_{0}, t_{1}\right),
$$

$\left[t_{0}, t_{1}\right)$ being the existence interval of solution $Z(t)$ (or $z(t)$ ).

If $U(t)$ is a fundamental matrix for the system $z^{\prime}=\left(I_{n} \otimes A(t)\right) z$, by the variation of constant formula ([3], Chapter II, section 2(8)),

$$
z(t)=U(t) U^{-1}\left(t_{0}\right) z_{0}+\int_{t_{0}}^{t} U(t) U^{-1}(s)\left(\left(B^{T}(s) \otimes I_{n}\right) z(s)+f(s, z(s))\right) d s,
$$

$t \in\left[t_{0} t_{1}\right)$.

From Lemma 8, we replace $U(t)=I_{n} \otimes X(t), X(t)$ being a fundamental matrix for the equation (4). After computation, it follows that

$$
\begin{gathered}
z(t)=\left(I_{n} \otimes\left(X(t) X^{-1}\left(t_{0}\right)\right)\right) z_{0}+ \\
+\int_{t_{0}}^{t}\left(I_{n} \otimes\left(X(t) X^{-1}(s)\right)\right)\left(\left(B^{T}(s) \otimes I_{n}\right) z(s)+f(s, z(s))\right) d s, t \in\left[t_{0}, t_{1}\right) .
\end{gathered}
$$

From hypothesis 2) and Theorem 1 [6], it follows that there exists a positive constant $K$ such that

$$
\left|I_{n} \otimes\left(\Psi(t) X(t) X^{-1}(s) \Psi^{-1}(s)\right)\right| \leq K,
$$

for all $t \geq s \geq 0$.

From (15), for $t \in\left[t_{0}, t_{1}\right)$, it follows that

$$
\begin{aligned}
& \left(I_{n} \otimes \Psi(t)\right) z(t)=\left(I_{n} \otimes\left(\Psi(t) X(t) X^{-1}\left(t_{0}\right) \Psi^{-1}\left(t_{0}\right)\right)\right)\left(I_{n} \otimes \Psi\left(t_{0}\right)\right) z_{0}+ \\
& +\int_{t_{0}}^{t}\left(I_{n} \otimes\left(\Psi(t) X(t) X^{-1}(s) \Psi^{-1}(s)\right)\right) \cdot \\
& \cdot\left(\left(B^{T}(s) \otimes \Psi(s)\right) z(s)+\left(I_{n} \otimes \Psi(s)\right) f(s, z(s))\right) d s, \text { and then, } \\
& \left\|\left(I_{n} \otimes \Psi(t)\right) z(t)\right\|_{\mathbb{R}^{n^{2}}} \leq K\left\|\left(I_{n} \otimes \Psi\left(t_{0}\right)\right) z_{0}\right\|_{\mathbb{R}^{n^{2}}}+ \\
& +K \int_{t_{0}}^{t}\left\|\left(B^{T}(s) \otimes \Psi(s)\right) z(s)+\left(I_{n} \otimes \Psi(s)\right) f(s, z(s))\right\|_{\mathbb{R}^{n^{2}}} d s, t \in\left[t_{0}, t_{1}\right) .
\end{aligned}
$$


From hypothesis 3) and Lemma 6, it follows that

$$
\begin{gathered}
\left\|\left(I_{n} \otimes \Psi(t)\right) f(t, z)\right\|_{\mathbb{R}^{n^{2}}}=\left\|\left(I_{n} \otimes \Psi(t)\right) \mathcal{V e c} F(t, Z)\right\|_{\mathbb{R}^{n^{2}}} \leq \\
\leq|\Psi(t) F(t, Z)| \leq \gamma(t)|\Psi(t) Z| \leq \\
\leq n \gamma(t)\left\|\left(I_{n} \otimes \Psi(t)\right) \mathcal{V} e c Z\right\|_{\mathbb{R}^{n^{2}}}=n \gamma(t)\left\|\left(I_{n} \otimes \Psi(t)\right) z\right\|_{\mathbb{R}^{n^{2}}},
\end{gathered}
$$

for $\mathrm{t} \in \mathbb{R}_{+}$and $\mathrm{z} \in \mathbb{R}^{n^{2}}$.

On the other hand,

$$
\begin{gathered}
\left\|\left(B^{T}(t) \otimes \Psi(t)\right) z\right\|_{\mathbb{R}^{n^{2}}}=\|\left(\left(B^{T}(t) \otimes I_{n}\right)\left(I_{n} \otimes \Psi(t)\right) z \|_{\mathbb{R}^{n^{2}}} \leq\right. \\
\leq\left|B^{T}(t) \otimes I_{n}\right|\left\|\left(I_{n} \otimes \Psi(t)\right) z\right\|_{\mathbb{R}^{n^{2}}},
\end{gathered}
$$

for $\mathrm{t} \in \mathbb{R}_{+}$and $\mathrm{z} \in \mathbb{R}^{n^{2}}$.

Now, from (16) we have

$$
\begin{gathered}
\left\|\left(I_{n} \otimes \Psi(t)\right) z(t)\right\|_{\mathbb{R}^{n^{2}}} \leq K\left\|\left(I_{n} \otimes \Psi\left(t_{0}\right)\right) z_{0}\right\|_{\mathbb{R}^{n^{2}}}+ \\
+K \int_{t_{0}}^{t}\left(\left|B^{T}(s) \otimes I_{n}\right|+n \gamma(s)\right)\left\|\left(I_{n} \otimes \Psi(s)\right) z(s)\right\|_{\mathbb{R}^{n^{2}}} d s, t \in\left[t_{0}, t_{1}\right)
\end{gathered}
$$

and therefore by Gronwall's inequality,

$$
\begin{gathered}
\left\|\left(I_{n} \otimes \Psi(t)\right) z(t)\right\|_{\mathbb{R}^{n^{2}}} \leq K\left\|\left(I_{n} \otimes \Psi\left(t_{0}\right)\right) z_{0}\right\|_{\mathbb{R}^{n^{2}}} . \\
\cdot e^{K \int_{t_{0}}^{t}\left(\left|B^{T}(s) \otimes I_{n}\right|+n \gamma(s)\right) d s} \leq \\
\leq K e^{n K\left(L+L^{\prime}\right)}\left\|\left(I_{n} \otimes \Psi\left(t_{0}\right)\right) z_{0}\right\|_{\mathbb{R}^{n^{2}}} \text {, for all } t \in\left[t_{0}, t_{1}\right) .
\end{gathered}
$$

This inequality shows that $t_{1}=+\infty$ and hence, the solution $z$ is defined on $\left[t_{0},+\infty\right)$. Therefore, it follows that the trivial solution of the system (14) is $I_{n} \otimes \Psi$ - uniformly stable on $\mathbb{R}_{+}$.

From Lemma 7 [6], it follows that the trivial solution of the equation (1) is $\Psi-$ uniformly stable on $\mathbb{R}_{+}$.

To prove that it is $\Psi$ - asymptotically stable, we must show that $\lim _{t \rightarrow \infty} \Psi(t) Z(t)=$ $O_{n}$.

For this, from the hypothesis 2) and Theorem 1, we have that $\lim _{t \rightarrow \infty} I_{n} \otimes \Psi(t) X(t)=$ $O_{\mathrm{n}}$.

From the hypotheses 3) and 4), given any $\varepsilon>0$, we can choose $t_{1} \geq t_{0}$ so large such that

$$
n K^{2} e^{n K\left(L+L^{\prime}\right)}\left\|\left(I_{n} \otimes \Psi\left(t_{0}\right)\right) z_{0}\right\|_{\mathbb{R}^{n^{2}}} \int_{t_{1}}^{\infty}(|B(t)|+\gamma(t)) d t<\frac{\varepsilon}{2} .
$$


From (15), for $t \geq t_{1}$, we have that

$$
\begin{aligned}
\left(I_{n} \otimes \Psi(t)\right) z(t)= & \left(I_{n} \otimes \Psi(t) X(t)\right) \cdot\left(I_{n} \otimes X^{-1}\left(t_{0}\right) \Psi^{-1}\left(t_{0}\right)\right) \cdot\left(I_{n} \otimes \Psi\left(t_{0}\right)\right) z_{0}+ \\
& +\int_{t_{0}}^{t_{1}}\left(I_{n} \otimes \Psi(t) X(t)\right)\left(I_{n} \otimes X^{-1}(s) \Psi^{-1}(s)\right) \cdot \\
& \cdot\left(\left(B^{T}(s) \otimes \Psi(s)\right) z(s)+\left(I_{n} \otimes \Psi(s)\right) f(s, z(s))\right) d s+ \\
& \quad+\int_{t_{1}}^{t}\left(I_{n} \otimes \Psi(t) X(t) X^{-1}(s) \Psi^{-1}(s)\right) \cdot \\
& \cdot\left(\left(B^{T}(s) \otimes \Psi(s)\right) z(s)+\left(I_{n} \otimes \Psi(s)\right) f(s, z(s))\right) d s
\end{aligned}
$$

and then

$$
\begin{gathered}
\left\|\left(I_{n} \otimes \Psi(t)\right) z(t)\right\|_{\mathbb{R}^{n^{2}}} \leq\left|I_{n} \otimes \Psi(t) X(t)\right| \cdot \\
\cdot\left[\left|I_{n} \otimes X^{-1}\left(t_{0}\right) \Psi^{-1}\left(t_{0}\right)\right|\left\|\left(I_{n} \otimes \Psi\left(t_{0}\right)\right) z_{0}\right\|_{\mathbb{R}^{n^{2}}}+\right. \\
\left.+\int_{t_{0}}^{t_{1}}\left(\left|I_{n} \otimes X^{-1}(s) \Psi^{-1}(s)\right| \cdot\left\|\left(B^{T}(s) \otimes \Psi(s)\right) z(s)+\left(I_{n} \otimes \Psi(s)\right) f(s, z(s))\right\|_{\mathbb{R}^{n^{2}}}\right) d s\right]+ \\
+n K^{2} e^{n K\left(L+L^{\prime}\right)}\left\|\left(I_{n} \otimes \Psi\left(t_{0}\right)\right) z_{0}\right\|_{\mathbb{R}^{n^{2}}} \int_{t_{1}}^{t}(|B(s)|+\gamma(s)) d s<\varepsilon,
\end{gathered}
$$

for all large $t$.

Thus, $\lim _{t \rightarrow \infty}\left(I_{n} \otimes \Psi(t)\right) z(t)=0$ and then, $\lim _{t \rightarrow \infty} \Psi(t) Z(t)=O_{n}$.

The trivial solution of (1) is $\Psi$ - asymptotically stable on $\mathbb{R}_{+}$.

The proof is now complete.

Remark. The part of Theorem relating to $\Psi$ - uniform stability is the Theorem 6, [6].

Theorem 6. Suppose that:

1). the hypothesis $(H)$ is satisfied;

2). the equation (3) is $\Psi$ - uniformly and asymptotically stable on $\mathbb{R}_{+}$;

3). the matrix function $F$ satisfies the inequality

$$
|\Psi(t) F(t, Z)| \leq \gamma(t)|\Psi(t) Z|
$$

for all $t \in \mathbb{R}_{+}$and for all $Z \in \mathbb{M}_{n \times n}$, where $\gamma: \mathbb{R}_{+} \longrightarrow \mathbb{R}_{+}$is a continuous function on $\mathbb{R}_{+}$such that $\int_{0}^{\infty} \gamma(t) d t<+\infty$;

Then, the trivial solution of the equation (1) is $\Psi$ - uniformly and asymptotically stable on $\mathbb{R}_{+}$.

Proof. It is similar to the proof of the above Theorem. 
Remark. The part of Theorem relating to $\Psi$ - uniform stability is the Theorem 5, [6].

Remark. The Theorems 5 and 6 generalize the Theorem 6, [3], Chapter III, Section 3.

If we require that the linear system (3) is only $\Psi$ - asymptotically stable on $\mathbb{R}_{+}$, then the above Theorems are no longer true. This is shown by above Example 2.

Example 5. Consider the equation (1) with

$$
\begin{gathered}
\mathrm{A}(\mathrm{t})=\left(\begin{array}{cc}
2 & 1 \\
-1 & 2
\end{array}\right), B(t)=\left(\begin{array}{cc}
-1 & 2 \\
-1 & -4
\end{array}\right), \\
F(t, Z)=\left(\begin{array}{cc}
\frac{1-\cos z_{1}}{1+t^{2}} & 0 \\
0 & e^{-t^{2}} \operatorname{arctg}_{4}
\end{array}\right),
\end{gathered}
$$

where $\mathrm{Z}=\left(\begin{array}{ll}z_{1} & z_{2} \\ z_{3} & z_{4}\end{array}\right)$

The fundamental matrices for the equations (4) and (5) are

$$
\begin{gathered}
X(t)=\left(\begin{array}{cc}
e^{2 t} \cos t & e^{2 t} \sin t \\
-e^{2 t} \sin t & e^{2 t} \cos t
\end{array}\right) \text { and } \\
Y^{T}(t)=\left(\begin{array}{cc}
2 e^{-2 t}-e^{-3 t} & e^{-3 t}-e^{-2 t} \\
2 e^{-2 t}-2 e^{-3 t} & 2 e^{-3 t}-e^{-2 t}
\end{array}\right)
\end{gathered}
$$

respectively.

Consider

$$
\Psi(t)=\left(\begin{array}{ll}
e^{-2 t} & \\
& e^{-2 t}
\end{array}\right)
$$

We have

$$
\Psi(t) X(t) X^{-1}(s) \Psi^{-1}(s)=\left(\begin{array}{cc}
\cos (t-s) & \sin (t-s) \\
-\sin (t-s) & \cos (t-s)
\end{array}\right)
$$

and

$$
Y^{T}(t)\left(Y^{T}(s)\right)^{-1}=\left(\begin{array}{cc}
2 e^{-2(t-s)}-e^{-3(t-s)} & e^{-3(t-s)}-e^{-2(t-s)} \\
2 e^{-2(t-s)}-2 e^{-3(t-s)} & 2 e^{-3(t-s)}-e^{-2(t-s)}
\end{array}\right) .
$$

From the Corollary of the above Theorem 1 and Theorem 1, [6], it follows that the equation (3) is $\Psi$ - uniformly and $\Psi$ - asymptotically stable on $\mathbb{R}_{+}$. 
Further, the function F satisfies a Lipschitz condition and

$$
|\Psi(t) F(t, Z)| \leq \frac{1}{1+t^{2}}|\Psi(t) Z|,
$$

for all $\mathrm{t} \in \mathbb{R}_{+}$and for all $\mathrm{Z} \in \mathbb{M}_{2 \times 2}$.

From these, it is easy to see that the function $F$ satisfies all the hypotheses of Theorem 6.

Thus, the trivial solution of the equation (1) is $\Psi$ - uniformly and $\Psi$ - asymptotically stable on $\mathbb{R}_{+}$.

Theorem 7. Suppose that:

a). the hypothesis $(H)$ is satisfied;

$b)$. there exist a continuous function $\varphi: \mathbb{R}_{+} \longrightarrow(0, \infty)$ with $\int_{0}^{\infty} \varphi(s) d s=+\infty$ and a positive constant $M$ such that the fundamental matrix $X$ of the equation (4) satisfies the condition

$$
\int_{0}^{t} \varphi(s)\left|\Psi(t) X(t) X^{-1}(s) \Psi^{-1}(s)\right| d s \leq M, \forall t \geq 0
$$

c). the matrix function $F$ satisfies the inequality

$$
|\Psi(t) F(t, Z)| \leq \gamma(t)|\Psi(t) Z|
$$

for all $t \in \mathbb{R}_{+}$and for all $Z \in \mathbb{M}_{n \times n}$, where $\gamma: \mathbb{R}_{+} \longrightarrow[0,+\infty)$ is a continuous function such that

$$
q=\sup _{t \geq 0} \frac{\left|B^{T}(t)\right|+n \gamma(t)}{\varphi(t)}<\frac{1}{M} .
$$

Then, the trivial solution of the equation (1) is $\Psi$ - asymptotically stable on $\mathbb{R}_{+}$.

Proof. As in the proof of Theorem 5, if $Z$ is the unique solution of (1) with $Z\left(t_{0}\right)=Z_{0}$, then, $z(t)=\operatorname{Vec} Z(t)$ satisfies $(15),\left[t_{0}, t_{1}\right)$ being the existence interval of solution $Z(t)$ (or $z(t)$ ).

From hypothesis b) and Theorem 2, it follows that there exists a positive constant K such that

$$
\left|I_{n} \otimes(\Psi(t) X(t))\right| \leq K e^{-M^{-1} \int_{1}^{t} \varphi(s) d s}, t \geq 0 .
$$

Consenquently,

$$
\lim _{t \rightarrow \infty} I_{n} \otimes(\Psi(t) X(t))=O_{n^{2}} .
$$

From (15), for $t \in\left[t_{0}, t_{1}\right)$ we have

$$
\left(I_{n} \otimes \Psi(t)\right) z(t)=\left(I_{n} \otimes \Psi(t) X(t)\right) \cdot\left(I_{n} \otimes X^{-1}\left(t_{0}\right) \Psi^{-1}\left(t_{0}\right)\right) \cdot\left(I_{n} \otimes \Psi\left(t_{0}\right)\right) z_{0}+
$$




$$
\begin{gathered}
+\int_{t_{0}}^{t}\left(I_{n} \otimes\left(\Psi(t) X(t) X^{-1}(s) \Psi^{-1}(s)\right)\right) . \\
\cdot\left(\left(B^{T}(s) \otimes \Psi(s)\right) z(s)+\left(I_{n} \otimes \Psi(s)\right) f(s, z(s))\right) d s,
\end{gathered}
$$

and then, as in the proof of Theorem 5,

$$
\begin{gathered}
\left\|\left(I_{n} \otimes \Psi(t)\right) z(t)\right\|_{\mathbb{R}^{n^{2}}} \leq K\left|I_{n} \otimes X^{-1}\left(t_{0}\right) \Psi^{-1}\left(t_{0}\right)\right|\left\|\left(I_{n} \otimes \Psi\left(t_{0}\right)\right) z_{0}\right\|_{\mathbb{R}^{n^{2}}}+ \\
\quad+\int_{t_{0}}^{t} \varphi(s)\left|\Psi(t) X(t) X^{-1}(s) \Psi^{-1}(s)\right| \cdot \\
\cdot \frac{\left|B^{T}(s)\right|+n \gamma(s)}{\varphi(s)} \cdot\left\|\left(I_{n} \otimes \Psi(s)\right) z(s)\right\|_{\mathbb{R}^{n^{2}}} d s \leq \\
\leq K\left|I_{n} \otimes X^{-1}\left(t_{0}\right) \Psi^{-1}\left(t_{0}\right)\right|\left\|\left(I_{n} \otimes \Psi\left(t_{0}\right)\right) z_{0}\right\|_{\mathbb{R}^{n^{2}}}+ \\
+q M \sup _{t_{0} \leq s \leq t}\left\|\left(I_{n} \otimes \Psi(s)\right) z(s)\right\|_{\mathbb{R}^{n^{2}}} \cdot
\end{gathered}
$$

From this, we have

$$
\sup _{t_{0} \leq s \leq t}\left\|\left(I_{n} \otimes \Psi(s)\right) z(s)\right\|_{\mathbb{R}^{n^{2}}} \leq \frac{K\left|I_{n} \otimes X^{-1}\left(t_{0}\right) \Psi^{-1}\left(t_{0}\right)\right|}{1-q M}\left\|\left(I_{n} \otimes \Psi\left(t_{0}\right)\right) z_{0}\right\|_{\mathbb{R}^{n^{2}}} .
$$

Thus, for $t \in\left[t_{0}, t_{1}\right)$,

$$
\left\|\left(I_{n} \otimes \Psi(t)\right) z(t)\right\|_{\mathbb{R}^{n^{2}}} \leq \frac{K\left|I_{n} \otimes X^{-1}\left(t_{0}\right) \Psi^{-1}\left(t_{0}\right)\right|}{1-q M}\left\|\left(I_{n} \otimes \Psi\left(t_{0}\right)\right) z_{0}\right\|_{\mathbb{R}^{n^{2}}} .
$$

This inequality shows that $t_{1}=+\infty$ and hence, the solution $z$ is defined on $\left[t_{0},+\infty\right)$. Therefore, it follows that the trivial solution of the equation (14) is $I_{n} \otimes \Psi$ - stable on $\mathbb{R}_{+}$. To prove that it is $I_{n} \otimes \Psi$ - asymptotically stable on $\mathbb{R}_{+}$, we must show further that $\lim _{\mathrm{t} \rightarrow \infty}\left(I_{n} \otimes \Psi(t)\right) z(t)=0$.

Let $\ell=\limsup _{t \rightarrow \infty}\left\|\left(I_{n} \otimes \Psi(t)\right) z(t)\right\|_{\mathbb{R}^{n^{2}}}$ and choose $\theta$ such that $q M<\theta<1$. If $\ell>0$, then $\left\|\left(I_{n} \otimes \Psi(t)\right) z(t)\right\|_{\mathbb{R}^{n^{2}}} \leq \frac{\ell}{\theta}$ for $t \geq t_{1}$ say.

From (17), it follows that for $t \geq t_{1}$,

$$
\begin{gathered}
\left\|\left(I_{n} \otimes \Psi(t)\right) z(t)\right\|_{\mathbb{R}^{n^{2}}} \leq\left|I_{n} \otimes \Psi(t) X(t)\right|\left\|\left(I_{n} \otimes X^{-1}\left(t_{0}\right)\right) z_{0}\right\|_{\mathbb{R}^{n^{2}}} \\
+\left|I_{n} \otimes \Psi(t) X(t)\right| \| \int_{t_{0}}^{t_{1}}\left(I_{n} \otimes X^{-1}(s)\right) \cdot\left(\left(B^{T}(s) \otimes I_{n}\right) z(s)+f(s, z(s)) d s \|_{\mathbb{R}^{n^{2}}}\right. \\
+q M \frac{\ell}{\theta} .
\end{gathered}
$$


Letting $t \rightarrow+\infty$, we get $\ell \leq q M \frac{\ell}{\theta}$, which is impossible. Therefore, $\ell=0$.

Now, from Lemma 7, it follows that the trivial solution of the equation (1) is $\Psi-$ asymptotically stable on $\mathbb{R}_{+}$.

The proof is now complete.

Remark. The part of Theorem relating to $\Psi$ - stability is the Theorem 8, [6].

Theorem 8. Suppose that:

a). the hypothesis $(H)$ is satisfied;

$b)$. there exists a continuous function $\varphi: \mathbb{R}_{+} \longrightarrow(0, \infty)$ with $\int_{0}^{\infty} \varphi(s) d s=+\infty$ and a positive constant $M$ such that the fundamental matrices $X$ and $Y$ for the equations (4) and (5) respectively satisfy the condition

$$
\int_{0}^{t} \varphi(s)\left|\left(Y^{T}(t)\left(Y^{T}(s)\right)^{-1}\right) \otimes\left(\Psi(t) X(t) X^{-1}(s) \Psi^{-1}(s)\right)\right| d s \leq M, t \geq 0
$$

c). the matrix function $F$ satisfies the inequality

$$
|\Psi(t) F(t, Z)| \leq \gamma(t)|\Psi(t) Z|
$$

for all $t \in \mathbb{R}_{+}$and for all $Z \in \mathbb{M}_{n \times n}$, where $\gamma: \mathbb{R}_{+} \longrightarrow[0,+\infty)$ is a continuous function such that

$$
q=\sup _{t \geq 0} \frac{\gamma(t)}{\varphi(t)}<\frac{1}{n M} .
$$

Then, the trivial solution of the equation (1) is $\Psi$ - asymptotically stable on $\mathbb{R}_{+}$.

Proof. It is similar to the proof of the above Theorem.

Remark. The part of Theorem relating to $\Psi-$ stability is the Theorem 7, [6].

Remark. The Theorems 7 and 8 generalize the Theorem 8, [3], Chapter III, Section 3.

\section{References}

[1] R. Bellman, Introduction to Matrix Analysis, McGraw-Hill Book Company, Inc. New York (translated in Romanian), 1960.

[2] L. Cesari, Un nuovo criterio di stabilita per le soluzioni delle equazioni differenziali lineari, Ann. Scuola Norm. Sup. Pisa, 2, 9, (1940), 163 - 186.

[3] W. A. Coppel, Stability and Asymptotic Behavior of Differential Equations, Heath, Boston, 1965. 
[4] A. Diamandescu, On the $\Psi-$ Asymptotic Stability of a Nonlinear Volterra IntegroDifferential System, Bull. Math. Soc. Sc. Math., Tome 46(94) No. 1 - 2, (2003), 39 - 60.

[5] A. Diamandescu, $\Psi$ - bounded solutions for a Lyapunov matrix differential equation, Electronic Journal of Qualitative Theory of Differential Equations, No. 17, 1 -11. URL: http://www.math.u-szeged.hu/ejqtde/, (2009.)

[6] A. Diamandescu, On $\Psi$ - stability of nonlinear Lyapunov matrix differential equations, Electronic Journal of Qualitative Theory of Differential Equations, No. 54, 1 - 18. URL: http://www.math.u-szeged.hu/ejqtde/, (2009)

[7] J. L. Massera and J. J. Schäffer, Linear differential equations and functional analysis, I, Ann. of math. 67, (1958), 517 - 573.

[8] M. S. N. Murty and B. V. Apparao, On two point boundary value problems for $\dot{X}=A X+$ XB, Ultra Science, 16(2)M, (2004), 223 - 227.

[9] M. S. N. Murty and B. V. Apparao, Two point boundary value problems for matrix differential equations, Journal of the Indian Math. Soc., Vol. 73, Nos. 1-2, (2006), 1-7.

[10] M. S. N. Murty and B. V. Apparao, Kronecker product boundary value problems - existence and uniqueness, Journal of Indian Acad. Mathematics, 25, No. 2, (2003), 383 - 394.

[11] M. S. N. Murty, B. V. Apparao, and G. Suresh Kumar, Controllability, observability and realizability of matrix Lyapunov systems, Bull. Korean Math. Soc., 43, No. 1, (2006), 149 159.

[12] K. N. Murty, K. R. Prasad, and P. V. S. Anand, Two point boundary value problem associated with Lyapunov type matrix difference system, Dyn. Syst. and Appl., 4, (1995), $205-214$

[13] M. S. N. Murty and G. Suresh Kumar, On $\Psi$-boundedness and stability of matrix Lyapunov systems, J. Appl. Math. Comput, 26: 67 - 84, (2008.)

[14] M. S. N. Murty and G. Suresh Kumar, On dichotomy and conditioning for two-point boundary value problems associated with first order matrix Lyapunov systems, J. Korean Math. Soc., 45, No. 5, (2008), 1361 - 1378.

[15] O. Perron, Die Stabilitätsfrage bei Differentialgleichungen, Math. Z., 32, 703 - 728., (1930.)

Aurel Diamandescu

University of Craiova,

Department of Applied Mathematics,

13, ”Al. I. Cuza" st., 200585 Craiova,

România

E-mail: adiamandescuecentral.ucv.ro

Received: 23.11.2011

Accepted: 10.05.2012 